the case of formaldehyde this electronic excitation has been shown unambiguously by Dieke and Kistiakowsky ${ }^{1}$ to be associated with a change of electric moment in the $y$-plane, that is, perpendicular to the $\mathrm{C}=\mathrm{O}$ axis and in the plane of the two hydrogen atoms. The excitation of the other aldehydes may be presumed to proceed in the same way. Mulliken ${ }^{2}$ has pointed out that such an electronic transition will appreciably affect the $\mathrm{C}-\mathrm{H}$ bonds, a conclusion which Norrish ${ }^{3}$ had reached previously on chemical grounds.

3. These considerations may be extended to the ketones $\begin{aligned} & \mathbf{R}^{1} \\ & \mathbf{R}^{2}\end{aligned} \mathbf{C}=0$, where our own preliminary observations on acetone showed ill-defined separations of about $1100 \mathrm{~cm}^{-1}$. The separations described by Bowen and Thompson ${ }^{4}$ are twice as large and irregular in magnitude ; moreover, the frequency $2900 \mathrm{~cm}^{-1}$ of the Raman line with which they compare these separations, is characteristic of valency vibrations involving hydrogen atoms, rather than those postulated under ( 1 ) above. It is, therefore, clear that further confirmation is required before these larger separations can be accepted as characteristic of the $>\mathrm{C}=\mathrm{O}$ group.

4. Striking results have been obtained with acrolein which (through the kindness of Prof. W. L. Bragg) we have examined with the 21-foot grating at Manchester. In all other aldehydes except formaldehyde, the fine structure is either too complex to be analysed at present, or too diffuse to be recorded in detail. The absorption spectrum of acrolein, however, shows several bands which have a fine structure rivalling those of the diatomic gases in simplicity and sharpness. These bands consist of sharp regularly spaced lines, of which as many as 20 may be seen in a range of less than $20 \mathrm{~A}$. They resemble the rotational lines of the $R$-branch of a diatomic molecule; but they present a remarkable anomaly, which does not appear to have been observed previously, since the moment of inertia of the ground state calculated on the assumption that the lines constitute single rotational series is different for the various bands, instead of being constant throughout.

C. P. SNow.

E. Eastwood

Laboratory of Physical Chemistry, Cambridge.

May 6.

1 Phys. Rev., 45, 4; 1934.

Trans. Farad. Soc., 26, 391 ; 1931.

- NATURE, 133, 571, April 14, 1934.

\section{Relation of Materials of the Cell Nucleus to the Lethal Action of Ultra-Violet Radiation}

Several workers have suggested the possible relationship of materials of the cell nucleus to the lethal action of ultra-violet radiation ${ }^{1-4}$, but there seems to have been no systematic study of the problem heretofore.

The lethal action of such radiation for cell life has been found to begin abruptly at about $2950 \mathrm{~A}$., and to continue for shorter wave-lengths ${ }^{5-11}$. By exposing such materials as thymus nucleic acid, adenine, uracil, etc., to ultra-violet radiation through various long-wave pass filters and studying their absorption before and after irradiation, we have found $2950 \mathrm{~A}$. to be approximately the longest wave-length effective in their destruction.
Absorption studies of uracil ${ }^{12}$, etc., in concentra. tions approximately that in which these substances appear in the cell nucleus, show them to have marked absorption in the ultra-violet shorter than $2900 \mathrm{~A}$. The long-wave leg of the absorption band rises steeply between $3000 \mathrm{~A}$. and $2900 \mathrm{~A}$., its position agreeing closely with the wave-length threshold for the lethal action of ultra-violet radiation on bacteria.

Finally, thymus nucleic acid, yeast nucleic acid, etc., have been found to have marked absorption maxima at about $2600 \mathrm{~A} \cdot .^{12,13}$. This agrees quite closely with the optimum wave-length region for the lethal action of ultra-violet as found by various workers ${ }^{8,8,11,14-17}$

These three types of results indicate that materials of the cell nucleus play an important part in the lethal action of ultra-violet for at least many kinds of micro-organisms.

When the rays shorter than about $2900 \mathrm{~A}$. are filtered out, the irradiation of nuclear compounds has been found to bring about absorption changes indicative of the formation of isomers or more com. plex compounds. The possibility that these products of long-wave irradiation may be growth promoting or cell-division promoting is being investigated.

Full details of these experiments will be published elsewhere.

JoHN R. LOOFBOUROW. Francis F. Heyroth.

Basic Science Research Laboratory, University of Cincinnati, Cincinnati, Ohio. May 7.

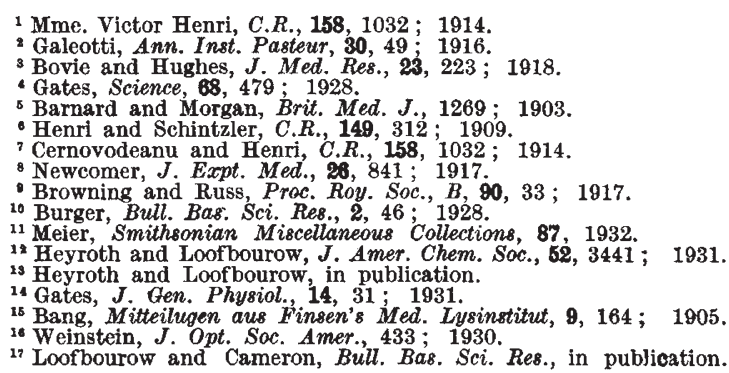

Hæmorrhages in Chicks Reared on Artificial Diets: a New Deficiency Disease

DURING work on the sterol metabolism of chicks ${ }^{1,2}$, it was observed that the animals often exhibited extensive internal hæmorrhages when they were fed a ration consisting of vitamin A-free casein 20 , Marmite 10 , salt mixture $4 \cdot 5$, starch $65 \cdot 5$ and varying amounts of cod liver oil concentrates. The hæmorrhages were subcutaneous or intramuscular on the breast, legs and wings, and were accompanied by certain pathological changes in the horny stratum of the gizzard.

The disease closely resembles scurvy, but it has been shown that vitamin $\mathrm{C}$ either in the form of lemon juice (up to 77 c.c. in 59 days) or pure ascorbic acid (given by mouth or subcutaneously) in very large doses was without the slightest influence on the occurrence of the symptoms. It has nothing to do with lack of vitamin $A, D, B_{1}, B_{2}$, 'fat' or cholesterol.

When chicks are fed a ration consisting entirely of cereals or seeds plus salts, the hæmorrhages fail 